\title{
Hochdurchsatzgenetik
}

\section{Funktion rätselhafter Retrons entschlüsselt}

JACOB BOBONIS, KARIN MITOSCH, ATHANASIOS TYPAS

EUROPÄISCHES LABORATORIUM FÜR MOLEKULARBIOLOGIE (EMBL), GENOME

BIOLOGY UNIT UND STRUCTURAL AND COMPUTATIONAL BIOLOGY UNIT, HEIDELBERG

\section{VAAM-Forschungspreis 2021}

Bacterial retrons are operons containing reverse transcriptases. Despite three decades of research, their physiological role remained mysterious. Recently, retrons have been uncovered as a widespread novel class of antiphage defense systems. We here discuss how high-throughput reverse genetics have enabled this discovery, illustrating the power such approaches have to illuminate the function of uncharacterized genes.

DOI: $10.1007 / \mathrm{s} 12268-021-1603-7$

(c) Die Autoren 2021

Schätzungsweise eine Billion verschiedener mikrobieller Arten und $10^{30}$ mikrobielle Zellen leben auf unserer Erde. Diese Biodiversität ist umso erstaunlicher, da auch innerhalb einer Art große genetische Unterschiede herrschen [1], sodass sich zwei Stämme derselben Bakterienart in ihrem Gengehalt extrem unterscheiden können. Für die meisten dieser unzähligen unterschiedlichen Gene ist keine Funktion bekannt. Selbst beim bestuntersuchten Mikroorganismus Escherichia coli K-12 ist für etwa ein Drittel der 4.500 Gene die Funktion unbekannt - und diese Zahl steigt nochmal signifikant an, wenn man das Pangenom von E. coli mit über 100.000 verschiedenen Genclustern einbezieht [2]. Bei weniger gut untersuchten Mikroorganismen ist diese Zahl noch deutlich höher [1].

Die Schwierigkeit bei der Aufklärung von Genfunktionen ist die Verknüpfung von Genen mit Phänotypen. Der Einsatz von neuen revers-genetischen Hochdurchsatzverfahren hat die Funktionszuweisung bei Genen bislang unbekannter Funktion revolutioniert. Tausende Gene verschiedener Mikroorganismen können hierbei direkt mit Phänotypen und/oder anderen Genen in Bezug gesetzt werden, was zum Verständnis ihrer Funktion beiträgt [3, 4]. Auf diese Wei- se gelang es uns kürzlich, die rätselhafte physiologische Rolle von Retrons aufzuklären. Sie wurden vor über 30 Jahren als erste bakterielle Operons beschrieben, die für reverse Transkriptasen (RTasen) codieren [5].

\section{Salmonella-Retron durch gestörte msDNA-Synthese aktiviert}

Zusätzlich zur RTase enthalten Retrons ein nicht codierendes RNA-Gen ( $m s r m s d$ ) und oft nicht charakterisierte akzessorische Gene unbekannter Funktion [5]. Retron-RTasen binden die zugehörige msrmsd-RNA und produzieren Hunderte Kopien von kurzen RNA/ DNA-Hybriden, die multicopy single-stranded DNA (msDNA), indem sie einen Teil der msrmsd-RNA in DNA umschreiben (Abb. 1A, [5]). Retrons sind weit verbreitet und werden horizontal zwischen prokaryotischen Genomen weitergegeben, sodass auch Stämme der gleichen Bakterienart unterschiedliche Retron-Typen aufweisen [5]. Trotz eines detaillierten mechanistischen Verständnisses, wie Retron-RTasen msDNAs mit unterschiedlichen Architekturen in den verschiedenen Bakterienstämmen produzieren - was häufig die Hilfe von weiteren Enyzmen wie der Ribonuklease $\mathrm{H}$ und anderen erfordert [5] - blieb ihre physiologische Rolle unklar.
Das lag vor allem daran, dass die Deletion der Retrons zu keinem besonderen Phänotyp führte. Erst als wir die Fitness aller Mutanten einer genomweiten Einzelgen-Deletionsbibliothek von Salmonella enterica ser. Typhimurium (STm) unter unterschiedlichen Bedingungen untersuchten, konnten wir zeigen, dass sich Mutanten mit einer gestörten msDNA-Produktion (hier vom RetronSen2) nicht mehr bei Temperaturen unter $25{ }^{\circ} \mathrm{C}[6]$ oder anaeroben Bedingungen vermehren können (Abb. 1B, [7]).

Um die Ursache hinter den Retron-assoziierten Phänotypen zu ermitteln, isolierten wir Supressormutanten, die unter kalten Bedingungen wieder wachsen können. Alle Mutationen befinden sich in einem Gen mit unbekannter Funktion, dem akzessorischen Gen im Retron direkt stromaufwärts des RTase-Gens [6]. Die durch die Mutationen verursachte Inaktivierung des Gens führt zum veränderten Phänotyp. Dies deutet darauf hin, dass die msDNA und/oder die RTase dafür nötig sind, einer möglichen Toxizität des Proteins, für das dieses Gen codiert, entgegenzuwirken. Indem wir das Gen in E. coli exprimierten, konnten wir die Toxizität des Genprodukts bestätigen, denn seine Expression hemmt das Wachstum der Bakterien. Dieser Effekt wird aufgehoben, wenn zusätzlich die Retron-Gene (die msDNA und die RTase) exprimiert werden. Wir nannten dieses akzessorische Gen deshalb rcaT (retron cold-anaerobic Toxin), da das Retron-Sen2 bemerkenswerte Ähnlichkeiten zu prokaryotischen Toxin/Antitoxin-Systemen aufweist.

\section{Retron-Sen2 erstes Beispiel für} dreiteiliges Toxin/Antitoxin-System

Prokaryotische Toxin/Antitoxin-Systeme sind binäre genetische Systeme aus einem Wachstumshemmer (Toxin) und einem zugehörigen Toxinhemmer (Antitoxin). Störungen des Antitoxins aktivieren das Toxin, das dann das bakterielle Wachstum hemmt, indem es essenzielle zelluläre Prozesse angreift [8]. Analog dazu wird RcaT im Retron-Sen2 aktiv, wenn die msDNA-Produktion gestört ist, was das Wachstum von STm 




$\Delta$ Abb. 1: Vom Phänotyp eines Retrons zu seiner physiologischen Rolle. A, Retrons produzieren eine multicopy single-stranded DNA (msDNA). Retrons bestehen aus einer reversen Transkriptase (RTase) und der nicht codierenden RNA ( $m s r m s d$ ) und enthalten oft nicht charakterisierte zusätzliche Gene [5] (z. B. RcaT im Retron Sen-2 [6]). Die RTase bindet an die hochstrukturierte msrmsdRNA und schreibt einen Teil davon in CDNA um. Das resultierende Hybrid aus RNA (orange) und DNA (pink) kann noch weiter zu einer einzelsträngigen DNA prozessiert werden (msDNA). B, Verknüpfung von Retrons mit Phänotypen mittels revers-genetischem Hochdurchsatzverfahren. Die Fitness von über 3.500 Einzelgen-Deletionsstämmen von Salmonella enterica serovar Typhimurium $(S T m)$ wurde unter über 500 verschiedenen Bedingungen quantifiziert. Die Deletion der Retron-Sen2-RTase oder der msrmsd-RNA schwächt die Bakterien unter kalten oder anaeroben Bedingungen [6, 7]. C, Retron-Sen2 ist ein Toxin/Antitoxin-System. Der Komplex aus RTase (blau) und msDNA (pink) des Retrons Sen2 bindet und inaktiviert das RcaT-Toxin (helllila). Wird die msDNA-Synthese oder die Intaktheit der RTase gestört, aktiviert dies das RcaT-Toxin (dunkellila) [6]. D, TIC/TAC, ein Hochdurchsatzansatz, um Toxin-Trigger und -Blocker zu finden. Plasmidbibliotheken, die einzelne Gene aus unterschiedlichen Stämmen exprimieren $\left(P_{\operatorname{tac}}-G e n 1-P_{\operatorname{tac}}-G e n N\right)$ werden mittels Konjugation auf Empfängerstämme übertragen, die die Plasmide $P_{B A D}$-Toxin/Antitoxin oder $\mathrm{P}_{B A D}$-Toxin tragen. Exprimiert man $\mathrm{P}_{B A D}$-Toxin/Antitoxin gemeinsam mit einem $P_{\text {tac }}$-Gen, das einen Toxin-Trigger enthält, kommt es zur toxininduzierten Wachstumshemmung (TAC); dahingegen wird die toxininduzierte Wachstumshemmung gelindert, wenn $P_{B A D}$-Toxin gemeinsam mit einem $P_{\text {tac }}$-Gen, das einen Toxin-Blocker enthält, exprimiert wird (TIC) [11]. E, Retrons schützen Bakterien vor Phagen. Phagenproteine (z. B. Dam, RecE) aktivieren Retron-Toxin/AntitoxinSysteme, indem sie das RTase-msDNA-Antitoxin außer Kraft setzen. Das aktivierte Retron-Toxin (dunkellila) kann dann das Wachstum des infizierten Bakteriums hemmen, womit indirekt die Weiterverbreitung des Phagen gestoppt wird (abortive Infektion, Abi). Als Antwort darauf enthalten Phagen auch Blocker-Proteine, die die Aktivität von Retron-Toxinen hemmen können.

unter kalten und anaeroben Bedingungen hemmt (Abb. 1C, [6]).

Toxin/Antitoxin-Systeme werden in verschiedene Typen eingeteilt, je nachdem wie das Antitoxin (RNA oder Protein) das Toxin hemmt [8]. Das Retron-Sen2 ist allerdings das erste Beispiel für ein dreiteiliges Toxin/ Antitoxin-System (msDNA-RcaT-RTase) sowie das erste Beispiel für einen DNAbasierten Wirkmechanismus des Antitoxins. rischen Gene auf $[9,10]$. Es ist noch unklar, ob andere akzessorische Gene in Retrons ähnliche Toxinfunktionen wie RcaT haben.

\section{Phagenproteine aktivieren oder hemmen Retron-Sen2}

Obwohl Retron-Sen2 wie ein Toxin/Antitoxin-System funktioniert, blieb seine physiologische Rolle weiterhin unklar, wie dies bei den meisten Toxin/Antitoxin-Systemen der Fall ist [8]. Um Hinweise auf die Funktion des Elements zu bekommen, versuchten wir, Proteine zu identifizieren, die die Toxizität von Retron-Sen2 auslösen oder blockieren. Dafür entwickelten wir einen neuartigen, auf reverser Genetik beruhenden, Hochdurchsatzansatz, den wir toxin inhibition/activation conjugation (TIC/TAC) nannten (Abb. 1D, [11]). TIC/TAC basiert auf der Quantifizierung, ob und wie effektiv Tausende von individuell exprimierten Genen das Toxin vom Antitoxin befreien (Trigger) oder die Aktivität des Toxins hemmen können (Blocker). Dazu verwendeten wir GenüberexpressionsBibliotheken, die auf Plasmiden einzeln jedes Gen von $E$. coli K-12 und E. coli B überexprimieren [11], und testeten sie in Stämmen, die entweder das Retron-Sen2-Toxin allein oder Toxin und Antitoxin zusammen exprimieren. Auf diese Weise identifizierten wir mehr als ein Dutzend Blocker und Trigger für das Retron-Sen2. Auffallend war dabei, dass viele der so identifizierten Faktoren einen eindeutigen Bezug zu Bakteriophagen aufwiesen [11].

Wir fanden heraus, dass Phagen-Trigger die Toxizität von RcaT aktivieren, indem sie das RTase-msDNA-Antitoxin durch direkte Wechselwirkung mit der msDNA inaktivieren. Beispielsweise aktiviert die ektopische Expression der Methyltransferase Dam oder der Exonuklease RecE (beide kommen häufig in Phagen vor [11]) die Toxizität von RcaT, indem entweder ein einzelnes Adenin in der msDNA methyliert oder die gesamte msDNA abgebaut wird. Andererseits blockiert die Überexpression kleiner Phagenproteine wie RacC (etwa 90 Aminosäuren lang) die Toxizität von RcaT vollständig. Bemerkenswerterweise befindet sich $\mathrm{racC}$ neben $\mathrm{recE}$ im RacProphagen-Genlocus; demnach können Blocker und Trigger gepaart in Phagengenomen vorkommen. Die Wechselwirkungen von Phagenproteinen mit Retron-Sen2 führten uns zu der Hypothese, dass Retrons während einer Phageninfektion eine Rolle spielen. Begleitstudien zu anderen Retron-Typen [10, 12] bestätigten, dass unterschiedliche 
Retrons Anti-Phagen-Aktivitäten aufweisen und als abortive Infektionssysteme wirken (Abb. 1E). Solche Systeme hemmen das Wachstum infizierter Zellen, was die Phagenvermehrung unterbindet und die verbleibende Bakterienpopulation schützt [8]. Retrons besitzen dabei eine außerordentliche Spezifität: Jedes Retron schützt gegen einige Phagen, aber nicht gegen andere. Wir nehmen an, dass diese Spezifität auf die jeweiligen Trigger und Blocker zurückzuführen ist, die der Phage mitbringt.

Retrons weisen eine außerordentliche Vielfalt auf und kommen in großer Zahl in prokaryotischen Genomen vor $[9,10]$. Auch wenn ihre physiologische Funktion nun bekannt ist, so bleiben dennoch viele Fragen offen: Wie erkennen verschiedene Retrons bestimmte Phagen, wie funktionieren ihre akzessorischen Gene, und wie können Phagen die Wirkung der Retrons umgehen? In den letzten Jahren wurde viel über den Sinn und Zweck der riesigen Anzahl von Toxin/ Antitoxin-Systemen spekuliert: Warum braucht Mycobacterium tuberculosis H37Rv über 80 Toxin/Antitoxin-Systeme vom Typ II [8]? Möglicherweise sind viele Toxin/Antitoxin-Systeme am Wettrüsten gegen Phagen beteiligt, und vielleicht werden bisher uncharakterisierte Teile vieler Phagengenome dafür eingesetzt, Toxin/Antitoxin-Systemen entgegenzuwirken. Revers genetische Ansätze wie unser neu entwickelter TIC/TACAssay werden eine entscheidende Rolle bei der Analyse dieses Wettrüstens spielen. Auf größerer Skala werden sie aber auch dazu beitragen, Licht in die faszinierende funktionale Vielfalt zu bringen, die drei Milliarden Jahre bakterielle Evolution hervorgebracht haben.

\section{Literatur}

[1] Almeida A, Aouacheria A, Arighi C et al. (2021) A unified catalog of 204,938 reference genomes from the human gut microbiome. Nat Biotechnol 39: 105-114

[2] Abram K, Udaondo Z, Bleker C et al. (2021) Mash-based analyses of Escherichia coli genomes reveal 14 distinct phylogroups. Commun Biol 4: 117

[3] Nichols RJ, Sen s, Choo YJ et al. (2011) Phenotypic landscape of a bacterial cell. Cell 144: 143-156

[4] Price MN, Wetmore KM, Waters RJ et al. (2018) Mutant phenotypes for thousands of bacterial genes of unknown function. Nature 557: 503-509

[5] Simon AJ, Ellington AD, Finkelstein ID (2019) Retrons and their applications in genome engineering. Nucleic Acids Res 47: 11007-11019

[6] Bobonis J, Mateus A, Pfalz B et al. (2020) Bacterial retrons encode tripartite toxin/antitoxin systems. bioRxiv, doi: https://doi.org/10.1101/2020.06.22.160168 [7] Elfenbein JR, Knodler LA, Nakayasu ES et al. (2015) Multicopy single-stranded DNA directs intestinal colonization of enteric pathogens. PLOS Genet 11: e1005472

[8] Harms A, Brodersen DE, Mitarai N, Gerdes K (2018)

Toxins, targets, and triggers: an overview of toxin-antitoxin biology. Mol Cell 70: 768-784

[9] Mestre MR, González-Delgado A, Gutiérrez-Rus LI et al. (2020) Systematic prediction of genes functionally associated with bacterial retrons and classification of the encoded tripartite systems. Nucleic Acids Res: 1-16
[10] Millman A, Bernheim A, Stokar-Avihail A et al. (2020) Bacterial retrons function in anti-phage defense. Cell 183: 1551-1561

[11] Bobonis J, Mitosch K, Mateus A et al. (2020) Phage proteins block and trigger retron toxin/antitoxin systems. bioRxiv, doi: https://doi.org/10.1101/2020.06.22.160242 [12] Gao L, Altae-Tran H, Böhning F et al. (2020) Diverse enzymatic activities mediate antiviral immunity in prokaryotes. Science 369: 1077-1084

Funding note: Open Access funding enabled and organized by Projekt DEAL. Open Access: Dieser Artikel wird unter der Creative Commons Namensnennung 4.0 International Lizenz veröffentlicht, welche die Nutzung, Vervielfältigung, Bearbeitung, Verbreitung und Wiedergabe in jeglichem Medium und Format erlaubt, sofern Sie den/die ursprünglichen Autor(en) und die Quelle ordnungsgemäß nennen, einen Link zur Creative Commons Lizenz beifügen und angeben, ob Änderungen vorgenommen wurden. Die in diesem Artikel enthaltenen Bilder und sonstiges Drittmaterial unterliegen ebenfalls der genannten Creative Commons Lizenz, sofern sich aus der Abbildungslegende nichs ander nach gesetzlichen Vorschriften erlaubt ist, ist für die oben aufgeführten Weiterverwendungen des Materials die Einwilligung des jeweiligen

Rechteinhabers einzuholen. Weitere Details zur Lizenz entnehmen Sie bitte der Lizenzinformation auf http://creativecommons.org/licenses/by/4.0/deed.de.

\section{Korrespondenzadresse:}

Dr. Athanasios Typas

Europäisches Laboratorium für Molekularbiologie (EMBL)

Genome Biology Unit

Meyerhofstraße 1

D-69117 Heidelberg

athanasios.typas@embl.de

www.embl.de/research/units/genome_biology/ typas/index.html

AUTOR
Athanasios Typas
Biochemiestudium, Universität Thessaloniki, Griechenland. 2006 Promotion an der
FU Berlin, anschließend Postdoc, University of California, San Francisco, USA. 2010
NIH K99/R00 Pathway to Independence Award. 2012 Humboldt Sofja Kovaleskaja
Award. 2018 Consolidator Grant des European Research Council. Seit 2011 Gruppen-
leiter und seit 2020 Senior Scientist der Genome Biology Unit am EMBL in Heidelberg.
2021 VAAM-Forschungspreis.

